\title{
Boden, ein kostbares Gut?
}

\section{Wir nutzen den Boden in der Schweiz nicht haushälterisch}

Boden erfüllt unverzichtbare Funktionen für das pflanzliche, tierische und menschliche Leben auf der Erde. Trotzdem «verbrauchen wir den Boden und schädigen ihn, als käme er täglich frisch auf den Tisch». ${ }^{1}$

- Seit 1950 hat sich die überbaute Fläche der Schweiz mehr als verdoppelt, und die Versiegelung meist fruchtbarster Böden durch Überbauung geht in einem Rhythmus von wenigstens 2000-3000 Hektaren pro Jahr weiter. ${ }^{2}$ Im Einzelfall mag dies zwar immer mit der Schaffung von Wohnraum, von Arbeitsplätzen oder mit anderen wirtschaftlichen und sozialen Argumenten begründet sein; die Versiegelungen summieren sich aber und sind irreversibel. Versiegelte Flächen stehen für die natürlichen Regelungs- und Ausgleichsfunktionen sowie als Lebensräume nicht mehr zur Verfügung.

- Die bisher in Städte und Dörfer strukturierte Siedlungslandschaft des schweizerischen Mittellandes ist im Begriff, sich in einen amorphen «Siedlungsbrei» aufzulösen. ${ }^{3}$ Die Einflußbereiche der verschiedenen Agglomerationen berühren und überlagern sich, so daß bald von einer «Stadt schweizerisches Mittelland» gesprochen werden kann. ${ }^{4}$ Die verbleibenden naturnahen Flächen sind zerschnitten und flächendeckend mit Immissionen belastet.

- Die in den letzten Jahren gestiegenen Bodenpreise und Mieten haben zwar dazu beigetragen, die teuer bezahlten Flächen «an Ort» sparsamer zu nutzen, die Wohnungsnachfrage wurde jedoch nicht wirksam gedämpft. Die hohen Preise verstärken den Druck zur Erweiterung der Bauzonen und zum Ausweichen an immer abgelegenere Standorte. Steigende Bodenpreise fördern - in Erwartung weiterer Preisanstiege - den Kapital$\mathrm{flu} ß$ in den Bodenmarkt und erschweren den $\mathrm{Zu}$ gang zur Bodennutzung für einkommensschwache Bevölkerungsgruppen.

- Über die Belastung der Böden mit Schadstoffen ist vieles noch unerforscht. Zwar weiß man nun, daß die Schwermetallbelastung der Böden außerhalb der eigentlichen Belastungsgebiete die
Richtwerte der Bodenschutzverordnung im allgemeinen nicht überschritten hat, ${ }^{5}$ die Belastung dauert jedoch an. Punktuell massive Richtwertüberschreitungen sind innerhalb der Belastungszonen bekannt, die bis zu $10 \%$ der Landesfläche ausmachen. Über die Belastung des Bodens mit organischen Schadstoffen weiß man noch wenig.

- Für die Zerstörung der Bodenstruktur, zunehmende Verdichtung und Verminderung des Bodenlebens in den letzten Jahrzehnten mehren sich die Anhaltspunkte; allerdings gibt es dafür kaum wissenschaftliche Beweise. ${ }^{6}$ Einig ist man sich darin, daß die heute praktizierten Bewirtschaftungsmethoden und engen Fruchtfolgen die Erhaltung und Wiederherstellung einer stabilen Bodenstruktur und eines aktiven Bodenlebens mehr behindern als fördern.

- Bodenerosion auf schweizerischen Ackerböden hat aufgrund von Beobachtungen in den letzten Jahrzehnten zugenommen. Die Messungen ergaben, daß etwa $10-40 \%$ der Ackerflächen von Bodenabtragsprozessen betroffen ist und $d a \beta$ auf etwa $5-10 \%$ der Ackerflächen das Erosionsgeschehen über der tolerierbaren Grenze liegt. ${ }^{7}$

- Die naturnahen Flächen im schweizerischen Mittelland sind seit den fünfziger Jahren auf fast die Hälfte geschrumpft. ${ }^{8}$ Diese Lebensräume der wildlebenden Flora und Fauna sind stark von der Art der Nutzung des Bodens betroffen. Je künstlicher, je intensiver und je einförmiger diese Nutzung ist, desto weniger naturnahe Flächen bleiben übrig, und desto geringer ist ihr ökologischer Wert.

Gesamthaft betrachtet betreiben wir Raubbau an der unvermehrbaren Lebensgrundlage Boden. Die Veränderungen erfolgen allerdings langsam und schrittweise und sind zum Teil schwer nachweisbar. Der Raum Schweiz befindet sich jedoch heute in einem Zustand der Überbauung, der großräumigen Verschmierung von Schadstoffen und der Armut an naturnahen Flächen, wie er noch nie dagewesen ist; und es ist keine Trendwende absehbar. ${ }^{9}$

Rudolf Häberli, Dr. sc. techn., Programmleiter des Nationalen Forschungsprogrammes "Boden", Liebefeld-Bern 


\section{Weshalb können wir uns diesen rücksichtslosen Umgang mit dem Boden (noch) leisten?}

An sich wissen wir, daß der Raum, der uns allen zur Verfügung steht, endlich ist, daß wir den Boden rücksichtsvoller nutzen sollten. Weshalb tun wir es nicht? Weshalb tun wir mehrheitlich das Gegenteil? Eine Antwort besteht darin, daß wir den Konsequenzen räumlich und zeitlich (noch) ausweichen können, daß wir auf die technische Mach- und Korrigierbarkeit vertrauen und daß wir andere persönliche und gesellschaftliche Prioritäten setzen. ${ }^{10}$

Räumliche Dimension: Die Entwicklungen im Verkehr und in der Kommunikation haben zusammen mit dem steigenden Wohlstand den Aktionsradius jedes einzelnen und der Wirtschaft kolossal erweitert. Tägliche Arbeitswegdistanzen von 100 Kilometern sind auch in der Schweiz keine Seltenheit mehr. Großflächige und wenig ertragskräftige Nutzungen, zunehmend auch störende oder unangenehme Nutzungen, können in Randgebiete oder ins Ausland verschoben werden. Solange wir wirtschaftlich stark sind, steht uns gewissermaßen die ganze Welt zur Nutzung zur Verfügung. Wenn wir die noch verbleibende einheimische Landwirtschaft auch aufgeben würden, so stünde genügend Raum zur Verfügung, um die Siedlung, die Anlagen für den Verkehr, den Sport, die Erholung usw. ungebremst während 1-2 weiteren Jahrhunderten wie in den vergangenen Jahrzehnten auszubauen.

Zeitliche Dimension: Wir sind mehrheitlich Städter geworden und haben uns von den Abläufen der Natur gelöst. Produktions- und Ferientermine sind wichtiger als Jahreszeiten und Witterungsverlauf. In der Wirtschaft läuft die Zeit immer schneller. Zeitvorsprung ist der Schlüssel für die Überlegenheit gegenüber der Konkurrenz. Die rasche Verfügbarkeit von Produktionsräumen ist denn auch wichtiger als andere Standortsfaktoren, insbesondere auch wichtiger als der dafür zu bezahlende Preis.11 Auch im privaten Bereich hat diese Entwicklung ihren Niederschlag gefunden. Man lebt den Augenblick, wenn nötig auf Kredit, mit einem Wechsel auf die Zukunft. In diesem Umfeld fällt es leicht, das vorsorgliche Denken zu verdrängen ebenso wie die effektiven Gefahren, die aber voraussichtlich die nächste oder die übernächste Generation treffen werden.

Technische Dimension: Die technische Entwicklung hat uns das tägliche Leben erleichtert. Sie ist in vielen Bereichen Grundlage für unseren Wohlstand. Die Technik wird sich weiter entwickeln, und wir werden weiter an der Verbesserung der von uns eingesetzten Technik arbeiten. Die Technik hat uns bisher geholfen, die wesentlichsten Umweltschäden nachträglich wieder zu beheben. ${ }^{12}$ Es ist bequem zu glauben, daß die Technik uns auch in Zukunft die Probleme lösen wird.

Gesellschaftliche und persönliche Ziele: Als Ziel ist haushälterische Bodennutzung nur eines - und zudem ein junges - unter zahlreichen weiteren gesellschaftlichen und persönlichen Zielen, selbst nur ein Teilziel innerhalb des umfassenden Umweltschutzes. Für zahlreiche Personen und Gruppen, vor allem auch in noch weniger entwickelten Regionen, ist die Hebung des materiellen Wohlstandes nach wie vor das vorrangige Ziel. Ein weiteres wichtiges Ziel ist die gerechtere Verteilung der Güter unter den Menschen und die Verbesserung der sozialen Sicherheit. In der Praxis läßt sich dies am ehesten erreichen, wenn der Wohlstand wächst. Es gibt deshalb immer Gründe, um andere Ziele wichtiger einzustufen als den Bodenschutz. ${ }^{13}$

\section{Und doch ist es nötig, unseren Boden haushälterisch zu nutzen}

Im Grunde genommen wissen wir, daß die Welt nicht größer geworden ist. Das Land, das wir in Randgebieten oder im Ausland für unsere Zwecke beanspruchen, steht nicht einfach zur Verfügung. Es wird nicht auf «Neuland» verschoben, sondern wir entziehen dort mit unseren ausgelagerten Ansprüchen schwächeren Nutzungen, konkret den globalen Regelungssystemen - Stichwort «Tropenwälder»-den wildlebenden Tieren und Pflanzen, ${ }^{1+}$ den schwächeren Bevölkerungsgruppen, den «Boden». Im Grunde genommen wissen wir, daß wir uns mit unserem Zeitverhalten in Widerspruch zu den Naturgesetzen begeben. Die Erkenntnisse im Bodenschutz lehren uns, daß die ständigen irreversiblen Veränderungen - auch wenn jede für sich geringfügig ist - letzlich zur Zerstörung unseres Lebensraumes führen. Für den Bodenschutz sind Jahre und selbst Generationen (zu) kurze Zeitspannen. Der Boden gleicht vorerst vieles aus, braucht dann aber auch Jahrhunderte oder gar Jahrtausende, um entstandene Schäden wieder zu regenerieren. ${ }^{15}$

Im Grunde genommen wissen wir auch, daß die Technik nicht alle Probleme lösen wird: Zwar gelingt es mit Sanierungstechniken, aus relativ beschränkten Volumen hochbelasteter Böden (alte Industriestandorte, Deponien, Unfallstellen) Schadstoffe wieder zu entfernen, allerdings nur mit viel Aufwand und Energieverbrauch. Dabei entsteht aber nicht wieder fruchtbare, belebte Erde, sondern bloß für den Menschen nicht mehr giftiges Material. ${ }^{16}$ Dispers über große Bodenflächen bzw. Bodenvolumen verteilte Schadstoffe können dagegen mit technischen Mitteln nicht mehr entfernt werden. Ausgestorbene Arten sind ohnehin nicht wiederherstellbar. 
Im Grunde genommen wissen wir, daß nicht alles gleichzeitig möglich ist, daß es abzuwägen, zu gewichten, immer wieder nach tragfähigen Kombinationen zu suchen gilt. Die drei Ziele - Wohlstand, Güterverteilung und Ressourcenerhaltung - stehen untereinander in einer gewissen Konkurrenz. Maximale Produktion geht oft leichter unter Mißachtung der gerechten Verteilung und der sozialen Sicherheit; eine hohe Produktion bei ausgebauter Verteilund sozialer Gerechtigkeit mißachtet oft die Ansprüche der Umwelt und der Natur.

\section{Haushälterische Bodennutzung ist möglich}

Möglichkeiten, den Boden haushälterisch zu nutzen, bestehen. Sie sind zum Teil auch bereits bekannt und in der Praxis erprobt.

Im Vordergrund stehen:

Siedlungsentwicklung nach innen: Anstatt am Stadtrand auf der grünen Wiese weiter und weiter hinauszubauen, sollen die Siedlungen im Innern erneuert und - in angepaßter und differenzierter Art verdichtet werden. Das Potential für eine Siedlungsentwicklung nach innen ist groß. Bereits eine $15-25 \%$ ige Ausnützung der Ausbaupotentiale in den Dach- und Kellergeschossen sowie in Nebengebäuden bestehender Bauten würde ausreichen, um den Bedarf von 300000 bis 500000 zusätzlichen Wohnungen bis zum Jahre $2010 \mathrm{zu}$ decken. Allein die zielgerichtete Nutzung von $5 \%$ der vorhandenen und für Dienstleistungsarbeitsplätze bestens geeigneten SBB-Areale würde genügen, um den absehbaren Flächenbedarf im Bereich Arbeitsplätze zu decken. ${ }^{17}$ Zudem besteht auf ehemaligen Industriearealen ein großes, schlecht genutztes Potential. Für diese Entwicklung und Erneuerung im Innern gibt es allerdings keine allgemeinen und einfachen Rezepte. ${ }^{18}$ Veränderung des Bestehenden ist Maßarbeit, entscheidend ist dabei die Qualität. Es braucht innovative Architekten und Unternehmer; es braucht einsichtige und kooperative Quartierbewohner, und es braucht aktive Behörden. Diese Behörden werben für eine umsichtige, ganzheitliche Bodennutzung, gehen mit eigener Tätigkeit beispielhaft voran und fördern erwünschte Lösungen durch Beratung und speditive Verfahren, durch Landpolitik und Landabtausch, durch Unterstützung und Beteiligung an Pilotprojekten.

Verminderung der Stoffeinträge: Emissionen müssen an der Quelle vermindert werden (durch verbesserte Technik, verminderten Verbrauch, Ersatz gefährlicher Stoffe usw.). Längerfristig ist das Ziel ein "Stoffflußgleichgewicht» (nicht mehr in den Boden hinein, als aus diesem durch Abbau, Entzug durch Pflanzen usw. auch wieder ausgetragen wird). ${ }^{19}$ Auch die.Landwirtschaft muß ihre Stoffeinträge ver- mindern. Neben der Reduktion überhöhter Tierbestände wird eine Lenkungssteuer für Mineraldünger vorgeschlagen..$^{20}$

Gesamtheitliche, multifunktionale Landwirtschaft: Die Landwirtschaft soll nicht bloß produzieren, sondern auch ihre übrigen Funktionen wiederum erfüllen, insbesondere die Regelung der Stoff- und Wasserkreisläufe, die Gewährleistung der Lebensräume für Tiere und Pflanzen sowie die Erhaltung und Gestaltung der Landschaft als Erholungsraum. Damit wird ihr ein ökologischer Leistungsauftrag erteilt, was die Umgestaltung der Entschädigung nach sich zieht: stufenweiser Abbau der Unterstützung über die Produktepreise und Ausbau der Abgeltung für allgemeine Leistungen mit Hilfe von Direktzahlungen. Beim einzelnen Betrieb bedeutet dies: angemessener Viehanteil, genügende Fruchtfolge, zurückhaltende Düngung und Pflanzenschutz, Verzicht auf gewisse Hilfsstoffe, Schaffung und Pflege naturnaher Landschaftselemente.

Erhaltung und Wiederherstellung naturnaher Flächen: Naturnahe Flächen müssen nicht bloß in ihrem heutigen Bestand erhalten, sondern erweitert, vernetzt und von äußeren Einflüssen entlastet werden, wenn wenigstens die noch bestehende Artenvielfalt erhalten werden soll. Die entsprechenden Bedürfnisse sind auf Bundes-, Kantons- und Gemeindeebene in Form von «landschaftlichen Entwicklungskonzepten» zu konkretisieren. Zur Verwirklichung sind die Instrumente des Natur- und Heimatschutzes und der Raumplanung einzusetzen, aber auch die neuen Mittel der Verträge mit Landwirten und anderen Landschaftspflegern. Die Verantwortung zur Erhaltung und Pflege der naturnahen Flächen und zur Übernahme der entsprechenden Kosten ist in diesem Sinne neu zu regeln.

\section{Haushälterische Bodennutzung: Nehmen wir die Aufgabe endlich ernst!}

Haushälterische Bodennutzung ist nötig, wenn wir die Tier- und Pflanzenarten, den künftigen Generationen den Lebensraum und uns selber eine gesunde und lebenswerte Umwelt erhalten wollen. Sie ist auch möglich; sie ist aber anspruchsvoller, arbeitsintensiver und konfliktträchtiger als das bloß individuell und kurzfristig ausgerichtete Handeln ohne weitere Rücksichtnahme.

Bodenschutz kommt nicht von selbst. Bodenschutz verursacht Kosten. Die Kosten verdichteter, qualitativ aufgewerteter Siedlungen sind höher als diejenigen von Neubauten auf der grünen Wiese. Die boden- und tiergerechte Nahrungsmittelproduktion ist aufwendiger. Auch die Erhaltung und Wiederherstellung naturnaher Flächen verursacht Kosten, die nicht dem Eigentümer oder Bewirtschafter, der dar- 
aus keinen Erlös erzielen kann, überbunden werden können.

Es gibt weder «Superlösungen» für haushälterische Bodennutzung noch handliche Rezepte. Denn vieles muß miteinander vereint, Neues entwickelt, Widersprüchliches gegeneinander abgewogen, tragfähige Kompromisse müssen gefunden werden.

Haushälterische Bodennutzung bedeutet die Verbindung von kreativem Denken, ${ }^{21}$ technischer Entwicklung, sorgsamem Bewahren, langfristiger und weiträumiger Ausrichtung, insbesondere aber das ständige Bemühen um das «richtige $\mathrm{Ma} ß$ ».

Unser Wissen verpflichtet uns, in diesem Sinne verantwortlich zu handeln.

\section{Anmerkungen}

1 E. Kopp: Der Boden und unsere Zeit (Referat Solothurn); in VLP Schriftenfolge Nr. 40, 1985, S. 6

${ }^{2}$ R. Häberli et al.: Boden-Los, Schlußbericht des nationalen Forschungsprogrammes "Boden»; in Vorbereitung

$3 \mathrm{H}$. Leuzinger et al.: Mehrfachnutzung des Bodens in Übergangsbereichen; Bericht 22 des NFP "Boden", 1988

${ }^{4} \mathrm{M}$. Bassand et al.: L'urbanisation: faits et perspectives; in: Les enjeux de l'urbanisation/Agglomerationsprobleme in der Schweiz; Peter Lang Verlag, Bern, 1988, S. $51 \mathrm{ff}$.

${ }^{5} \mathrm{H}$. Vogel et al.: Schwermetallgehalte in den Böden der Schweiz; Bericht 40 des NFP "Boden", 1990

6 Über die Bodenfauna müssen vorerst Grundlagenuntersuchungen durchgeführt werden. Vgl. dazu: W. Matthey et al.: Invertébrés bioindicateurs de la qualité des sols agricoles; Bericht 56 des NFP «Boden", 1990

7 T. Mosimann et al.: Bodenerosion im schweizerischen Mittelland; Bericht 51 des NFP "Boden", 1990

$8 \mathrm{M}$. Broggi, H. Schlegel: Mindestbedarf an naturnahen Ausgleichsflächen in der Kulturlandschaft; Bericht 31 des NFP "Boden", 1989

${ }^{9}$ R. Häberli et al., op. cit.

${ }^{10}$ Es gibt allerdings Beispiele aus der Geschichte - so bis ins 18. Jahrhundert in der Zeit des "Ancien Régime" -, wo wesentlich strengere Nutzungsvorschriften als heute den Umgang mit dem Boden regelten. Sie wurden akzeptiert, weil praktisch jede Generation die Folgen ungenügender Ressourcen am eigenen Leibe erfuhr; Ursachen und Konsequen- zen fielen räumlich und zeitlich zusammen (W. Thut, Ch. Pfister: Haushälterischer Umgang mit Boden, Erfahrungen aus der Geschichte; Bericht 7 des NFP «Boden», 1986).

11 M. Rossi, C. Kopreinig Guzzi: Industrie, territoire, marché immobilier; Bericht 30 des NFP "Boden", 1989

12 Allerdings haben uns auch erst die mechanischen, chemischen und biologischen Erfindungen, insbesondere aber die Erschließung billiger Energie, dazu befähigt, die Natur und den Boden radikal zu dominieren, sie in noch nie gekanntem Ausmaße für unsere Zwecke auszubeuten, ihr in noch nie gekanntem Maße Schäden zuzufügen (vgl. dazu W. Thut und Ch. Pfister, op. cit.; sowie: H. Ruh et al.: Ethik und Boden; Bericht 52 des NFP "Boden", 1990)

13 Befragungen lassen zwar eine Zustimmung der Bevölkerung zum Prinzip der haushälterischen Bodennutzung erkennen; dieses geschärfte Problembewußtsein führt indessen nicht zu einem anderen Verhalten. Der einzelne hat gelernt, mit der Diskrepanz von rationalem Bewußtsein und persönlichem Handeln zu leben (P. Farago, M. Peters: Einstellungen zum Bodensparen, Bericht 48 des NFP “Boden», 1990)

${ }^{14}$ Allein in der Schweiz sind zwischen 30 und $70 \%$ der Pflanzen- und Tierarten in ihrem Überleben bedroht (M. Broggi und H. Schlegel, op. cit., S. 22)

${ }^{15}$ Zum Beispiel ist die natürliche Bodenneubildung äußerst gering. Sie beträgt im schweizerischen Mittelland höchstens $0,1 \mathrm{~mm} / \mathrm{Jahr}$ (T. Mosimann et al.: Erosionsbekämpfung in Akkerbaugebieten, Themenbericht des NFP “Boden", in Vorbereitung)

16 F. Arendt et al. (Hrsg.): Altlastensanierung '90; Dritter Internationaler KfK TNO-Kongreß über Altlastensanierung, Karlsruhe; Kluwer, Dordrecht, 1990

${ }^{17} \mathrm{Ch}$. Gabathuler: Siedlungsbegrenzung Schweiz; Bericht 57 des NFP “Boden», 1990

${ }^{18}$ A. Herczog, E. Hubeli: Qualifizierte Verdichtung in städtischen Außenquartieren; Bericht 25 des NFP "Boden», 1988

19 Um zu einem Gleichgewicht der Stoffflüsse im Boden zu gelangen, müßten beispielsweise die Phosphordüngung auf rund die Hälfte und die Schwermetalleinträge auf einen Zehntel der heutigen Werte reduziert werden (B. von Steiger, P. Baccini: Regionale Stoffbilanzierung von landwirtschaftlichen Böden mit meßbarem Ein- und Austrag; Bericht 38 des NFP “Boden", 1990)

20 A. Bidaux, U. Gantner: Comment freiner l'emploi des engrais minéraux et des pesticides en agriculture? Bericht 50 des NFP "Boden", 1990

21 Vgl. dazu J. Heeb et al.: Denkansätze und Handlungskonzepte für den Bodenschutz in der Schweiz; Bericht 55 des NFP «Boden", 1990 